\title{
Association of Post-Operative Radiotherapy With Survival in Resected N2 Non-Small Cell Lung Cancer Patients with Chemotherapy
}

\author{
Yuan Zeng \\ The First Affiliated Hospital of guangzhou Medical University \\ Jun Liu \\ The First Affiliated Hospital of guangzhou Medical University \\ Minghui Wan \\ The First Affiliated Hospital of guangzhou Medical University \\ Qiwen Li \\ Sun Yat-sen University Cancer Center \\ Hui Liu \\ Sun Yat-sen University Cancer Center \\ Fei Cui \\ The First Affiliated Hospital of guangzhou Medical University \\ Zhexue Hao \\ The First Affiliated Hospital of guangzhou Medical University \\ Wei Wang \\ The First Affiliated Hospital of guangzhou Medical University \\ Long Jiang \\ The First Affiliated Hospital of guangzhou Medical University \\ Wenhua Liang ( $\square$ liangwh1987@163.com) \\ The First Affiliated Hospital of guangzhou Medical University \\ Jianxing He \\ The First Affiliated Hospital of guangzhou Medical University
}

\section{Research}

Keywords: postoperative radiotherapy, pathologic N2 Non-Small Cell Lung Cancer, nomogram

Posted Date: July 7th, 2021

DOI: https://doi.org/10.21203/rs.3.rs-663176/v1

License: (c) (i) This work is licensed under a Creative Commons Attribution 4.0 International License. Read Full License 


\section{Abstract}

Backgroup

The current staging system for completely resected pathologic N2 non-small-cell lung cancer (NSCLC) treated with chemotherapy is not suitable for predicting those patients most likely to benefit or not from post-operative radiotherapy (PORT). This study aimed to construct a survival prediction model that will enable individualized predictions of the net survival difference of PORT.

Methods

A total of 3094 cases between 2002 and 2014 were extracted from the Surveillance, Epidemiology, and End Results databases. Patient characteristics were included as covariates, and their association for overall survival (OS) with and witout PORT was assessed. Externally validate data of 602 patients were included from China.

Results

Age, gender, examined lymph node, positive lymph node, tumor size, extent of surgery, and visceral pleural invasion were significantly associated with OS $(\mathrm{P}<.05)$. The two nomograms were developed based on clinical variables to estimate an individual's net survival difference attributable to PORT. The calibration curve for OS showed great agreement between prediction by survival prediction model and actual observation. In the training cohort, the C-index for OS was 0.619 (95\% Cl, 0.598-0.641) in the PORT group and 0.627 (95\% Cl, 0.605-0.648) in the non-PORT group. We found that PORT could improve OS $(\mathrm{HR}, 0.861 ; P=0.044)$ for patients with a positive PORT net survival difference.

Conclusions

We established a practical survival prediction model that can be used to make individualized estimate of the net survival difference of PORT and without PORT in patients with completely resected N2 NSCLC, treated with chemotherapy.

\section{Background}

The most generally diagnosed cancer is lung cancer, which is the leading cause of cancer based death (18.4\% of total cancer deaths) worldwide.[1] Patients with resected pathologic N2 non-small-cell lung cancer (NSCLC) are a high risk group for regional recurrence and metastasis even with complete resection [2]. Adjuvant chemotherapy is now recommended as standard for those patients according to the NCCN (National Comprehensive Cancer Network) guidelines.[3] Two large databases including SEER [4] and NCBD [5] suggest that post-operative radiotherapy (PORT) can improve survival for patients with resected pathologic N2 NSCLC. Some meta-analysis studies [6, 7] also demonstrated a benefit of PORT in N2 nodal disease. However, there are also articles suggesting that PORT has no significant effect on survival. [8-10] Furthermore, the NCCN guidelines are themselves ambiguous [3]. Therefore, the role of PORT in completely resected N2 NSCLC patients is still highly controversial. Currently, physicians have little evidence to suggest that PORT will be beneficial to their patients. Moreover, the current staging system of completely resected pathologic N2 NSCLC treated with chemotherapy is not sufficient for identifying the patients who may most likely benefit or not from PORT. Consequently, there it remains necessary to develop a survival model exploring the potential individual difference of PORT.

In this study, we aim to develop a survival prediction model to calculate the probable overall survival(OS) differences of PORT and without PORT for patients with completely resected pathologic N2 NSCLC treated with chemotherapy.

\section{Methods}

\section{Patient Selection Criteria}

Information for patients with completely resected pathologic N2 NSCLC treated with chemotherapy between 2002 and 2014 was extracted from the Surveillance, Epidemiology, and End Results (SEER) database (http://seer.cancer.gov/). The inclusion criteria contained the following: pathologically confirmed primary N2 NSCLC between January 2002 and December 2014; completely resected history of lobectomy or pneumonectomy; treatment with chemotherapy; and only one malignant primary lesion. The exclusion criteria were as follows: distant metastasis; invasion of any of the following structures: heart, great vessels, trachea, diaphragm, mediastinum, recurrent laryngeal nerve, carina, vertebral body, and esophagus; Preoperative radiotherapy; radioactive implants; radioisotopes; no information on extracted data.

An external validation cohort who met the inclusion and exclusion criteria was presented to analyze the applicability of the model. The cohort composed of 602 patients between 2009 and 2014 from the First Affiliated Hospital of Guangzhou Medical University and Collaborative Innovation Center for Cancer Medicine of Sun Yat-sen University, China.

The baseline demographics for the patients (age, sex, and race), characteristics of tumors (size, location, differentiation grade and histological type), examined lymph node, positive lymph node, extent of surgery and VPI (visceral pleural invasion) were gathered from the SEER database. The 
TNM category was based on the IASLC 8th edition staging system. [11] PORT was categorized as received or not received.

\section{Construction of the Nomogram}

In the training set for PORT and non-PORT groups, OS was predicted by the Kaplan-Meier method and analyzed applying the log-rank test. Multivariable Cox proportional hazard regression was applied to identify independent prognostic factors. On the basis of the significant independent factors of two groups, nomograms were formulated by R 3.5.3 with the rms and survival packages.[12] Rms is the package that goes along with the book Regression Modeling Strategies. All survival models were constructed using the rms R library by Harrell (http://cran.rproject.org/web/packages/rms).

\section{Validation and Calibration of the Nomogram}

The model was subjected to 1,000 bootstrap resamples for internal validation in the training cohort and external validation with the cohort from the Chinese Institute. Calibration for one, three, and five year OS was determined by comparing the predicted survival with the observed survival on 1000 bootstrap resamples. The ability to discriminate was determined using the concordance index (C-index). The values of the $\mathrm{C}$-index ranged from 0.5 to 1.0, and higher C-index values suggested a better outcome with the nomogram.[13] The C-index for two different models were compared using the methods previously described [14].

\section{Statistical Analyses}

The chi-square test was applied to examine the statistical significance of the differences for the clinical variables between the PORT and non-PORT groups. OS was calculated using the Kaplan-Meier method and compared by applying the log-rank test. The independent prognostic factors were identified using the multivariate cox proportional hazard regression. The hazard ratio (HR) and corresponding 95\% confidence interval (CI) were determined. Nomograms were developed using the rms package of R version 3.5.3. All statistical analyses were performed by SPSS 22.0 , and $\mathrm{P}<$ 0.05 was regarded statistically significant.

\section{Results}

\section{Clinical Characteristics}

A total of 3094 patients with completely resected pathologic N2 NSCLC and treated with chemotherapy derived from the SEER database (Figure S1), and 602 patients from a multicenter hospital in China met the inclusion criteria. The demographics and clinicopathological characteristics assessed for the training cohort and external validation cohort are listed in Table 1. The median interquartile range (IQR) and follow-up times on OS were 27 months $(13,52)$ and 36 months $(22,49)$ for the training cohort and external validation cohort, respectively. 
Table 1

Demographics and Clinicopathologic Characteristics of the Training and External Validation Cohort.

\begin{tabular}{|c|c|c|c|c|c|c|c|c|c|c|}
\hline \multirow[b]{3}{*}{ Characteristic } & \multicolumn{5}{|c|}{ Training cohort } & \multicolumn{5}{|c|}{ External Validation cohort } \\
\hline & \multicolumn{2}{|c|}{ PORT $(n=1519)$} & \multicolumn{3}{|c|}{ NO-PORT ( $n=1575)$} & \multicolumn{2}{|c|}{ PORT $(n=69)$} & \multicolumn{3}{|c|}{ NO-PORT (n = 533) } \\
\hline & No. & $\%$ & No. & $\%$ & $\mathrm{P}$ & No. & $\%$ & No. & $\%$ & $\mathrm{P}$ \\
\hline Age & & & & & 0.001 & & & & & 0.127 \\
\hline$<60$ & 517 & 34 & 495 & 31.4 & & 47 & 68.1 & 295 & 55.3 & \\
\hline $60-70$ & 594 & 39.1 & 558 & 35.4 & & 17 & 24.6 & 177 & 33.2 & \\
\hline$\geq 70$ & 408 & 26.9 & 522 & 33.1 & & 5 & 7.2 & 61 & 11.4 & \\
\hline Gender & & & & & 0.272 & & & & & 0.25 \\
\hline Male & 734 & 48.3 & 730 & 46.3 & & 45 & 65.2 & 309 & 58 & \\
\hline Female & 785 & 51.7 & 845 & 53.7 & & 24 & 34.8 & 224 & 42 & \\
\hline Race & & & & & 0.674 & & & & & \\
\hline White & 1236 & 81.4 & 1285 & 81.6 & & - & - & - & - & \\
\hline Black & 138 & 9.1 & 152 & 9.7 & & - & - & - & - & \\
\hline Other & 145 & 9.5 & 138 & 8.8 & & - & - & - & - & \\
\hline Location & & & & & 0.732 & & & & & 0.856 \\
\hline Upper & 875 & 57.6 & 896 & 56.9 & & 31 & 44.9 & 258 & 48.4 & \\
\hline Middle & 75 & 4.9 & 72 & 4.6 & & 7 & 10.1 & 41 & 7.7 & \\
\hline Lower & 516 & 34 & 548 & 34.8 & & 28 & 40.6 & 206 & 38.6 & \\
\hline other & 53 & 3.3 & 59 & 3.7 & & 3 & 4.3 & 28 & 5.3 & \\
\hline Examined lymph node & & & & & 0.035 & & & & & 0.837 \\
\hline $0-9$ & 696 & 45.8 & 649 & 41.2 & & 7 & 10.1 & 46 & 8.6 & \\
\hline $10-15$ & 404 & 26.6 & 453 & 28.8 & & 13 & 18.8 & 114 & 21.4 & \\
\hline$\geq 16$ & 419 & 27.6 & 473 & 30 & & 49 & 71 & 373 & 70 & \\
\hline Positive lymph node & & & & & 0.007 & & & & & 0.963 \\
\hline $1-3$ & 876 & 57.7 & 995 & 63.2 & & 26 & 37.7 & 210 & 39.4 & \\
\hline $4-9$ & 531 & 35 & 478 & 30.3 & & 28 & 40.6 & 211 & 39.6 & \\
\hline$\geq 10$ & 112 & 7.4 & 102 & 6.5 & & 15 & 21.7 & 112 & 21 & \\
\hline \multicolumn{11}{|l|}{ Tumor size } \\
\hline$\leq 3 \mathrm{~cm}$ & 715 & 47.1 & 719 & 45.7 & 0.305 & 30 & 43.5 & 259 & 48.6 & 0.524 \\
\hline$>3$ to $5 \mathrm{~cm}$ & 522 & 34.4 & 525 & 33.3 & & 29 & 42 & 183 & 34.3 & \\
\hline$>5$ to $7 \mathrm{~cm}$ & 187 & 12.3 & 230 & 14.6 & & 5 & 7.2 & 58 & 10.9 & \\
\hline$>7 \mathrm{~cm}$ & 95 & 6.3 & 101 & 6.4 & & 5 & 7.2 & 33 & 6.2 & \\
\hline Extent of surgery & & & & & 0.01 & & & & & 0.3 \\
\hline Lobectomy & 1385 & 91.2 & 1392 & 88.4 & & 68 & 98.6 & 512 & 96.1 & \\
\hline Pneumonectomy & 134 & 8.8 & 183 & 11.6 & & 1 & 1.4 & 21 & 3.9 & \\
\hline Differentiation grade & & & & & 0.834 & & & & & 0.126 \\
\hline Grade I & 72 & 4.7 & 83 & 5.3 & & 1 & 1.4 & 17 & 3.2 & \\
\hline Grade II & 633 & 41.7 & 663 & 42.1 & & 33 & 47.8 & 289 & 54.2 & \\
\hline Grade III or IV & 713 & 46.9 & 733 & 46.5 & & 19 & 27.5 & 158 & 29.6 & \\
\hline
\end{tabular}




\begin{tabular}{|c|c|c|c|c|c|c|c|c|c|}
\hline \multirow[b]{3}{*}{ Unknown } & \multicolumn{4}{|c|}{ Training cohort } & \multicolumn{5}{|c|}{ External Validation cohort } \\
\hline & \multicolumn{2}{|c|}{ PORT $(n=1519)$} & \multicolumn{2}{|c|}{ NO-PORT $(n=1575)$} & \multicolumn{2}{|c|}{ PORT $(n=69)$} & \multicolumn{2}{|c|}{ NO-PORT ( $n=533$ ) } & \\
\hline & 101 & 6.6 & 96 & 6.1 & 16 & 23.2 & 69 & 12.9 & \\
\hline Histology & & & & & & & & & 0.007 \\
\hline SC & 273 & 18 & 305 & 19.4 & 23 & 33.3 & 93 & 17.4 & \\
\hline Adenocarcinoma & 1028 & 67.7 & 1038 & 65.9 & 42 & 60.9 & 405 & 76 & \\
\hline Others & 218 & 14.4 & 232 & 14.7 & 4 & 5.8 & 35 & 6.6 & \\
\hline VPI & & & & & & & & & 0.968 \\
\hline Yes & 537 & 35.4 & 522 & 33.1 & 34 & 49.3 & 264 & 49.5 & \\
\hline No & 982 & 64.6 & 1053 & 66.9 & 35 & 50.7 & 269 & 50.5 & \\
\hline
\end{tabular}

\section{Independent Prognostic Factors in the Training Cohort}

The survival analysis using the log-rank test found no significant differences in OS (HR=1.006, 95\% Cl: 0.915 to 1.106; P = 0.9) between the PORT and non-PORT groups (Fig. 1a). Results from the multivariate regression model are listed in Table 2. For patients with PORT, the multivariate analyses indicated that age $(P<0.001)$, gender $(P=0.011)$, examined lymph node $(P<0.001)$, positive lymph node $(P<0.001)$, tumor size $(P=$ 0.037), extent of surgery $(P<0.032)$, and differentiation grade $(P=0.001)$ are independent prognostic factors for OS. For patients in the non-PORT group, the multivariate analyses indicated that age $(P<0.001)$, gender $(P<0.001)$, examined lymph node $(P=0.013)$, positive lymph node $(P<$ $0.001)$, tumor size $(P=0.005)$, and VPI $(P=0.046)$ are independent prognostic factors for $O S$. 
Table 2

Multivariate Cox Regression Analysis of Factors Associated With Overall Survival.

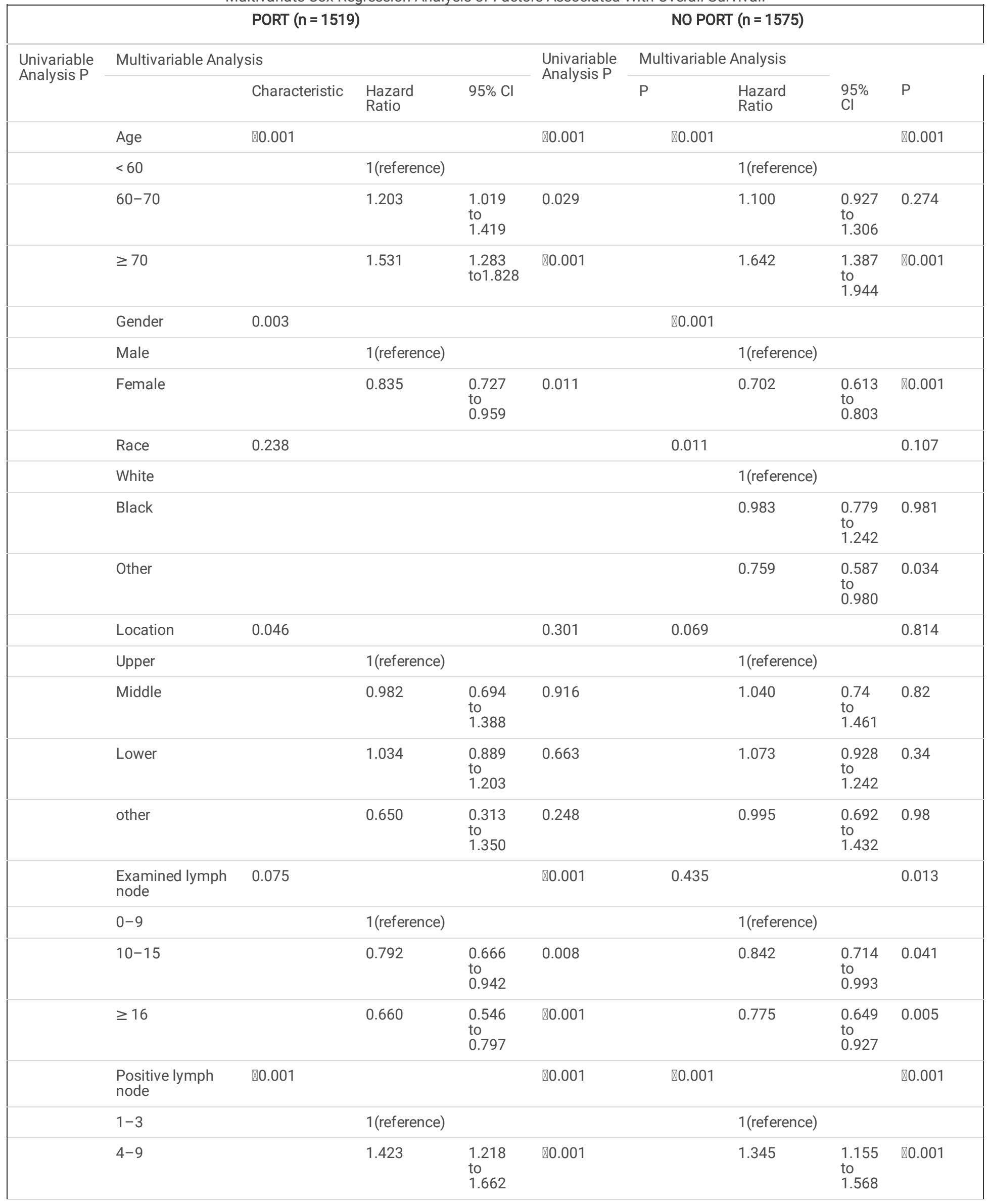




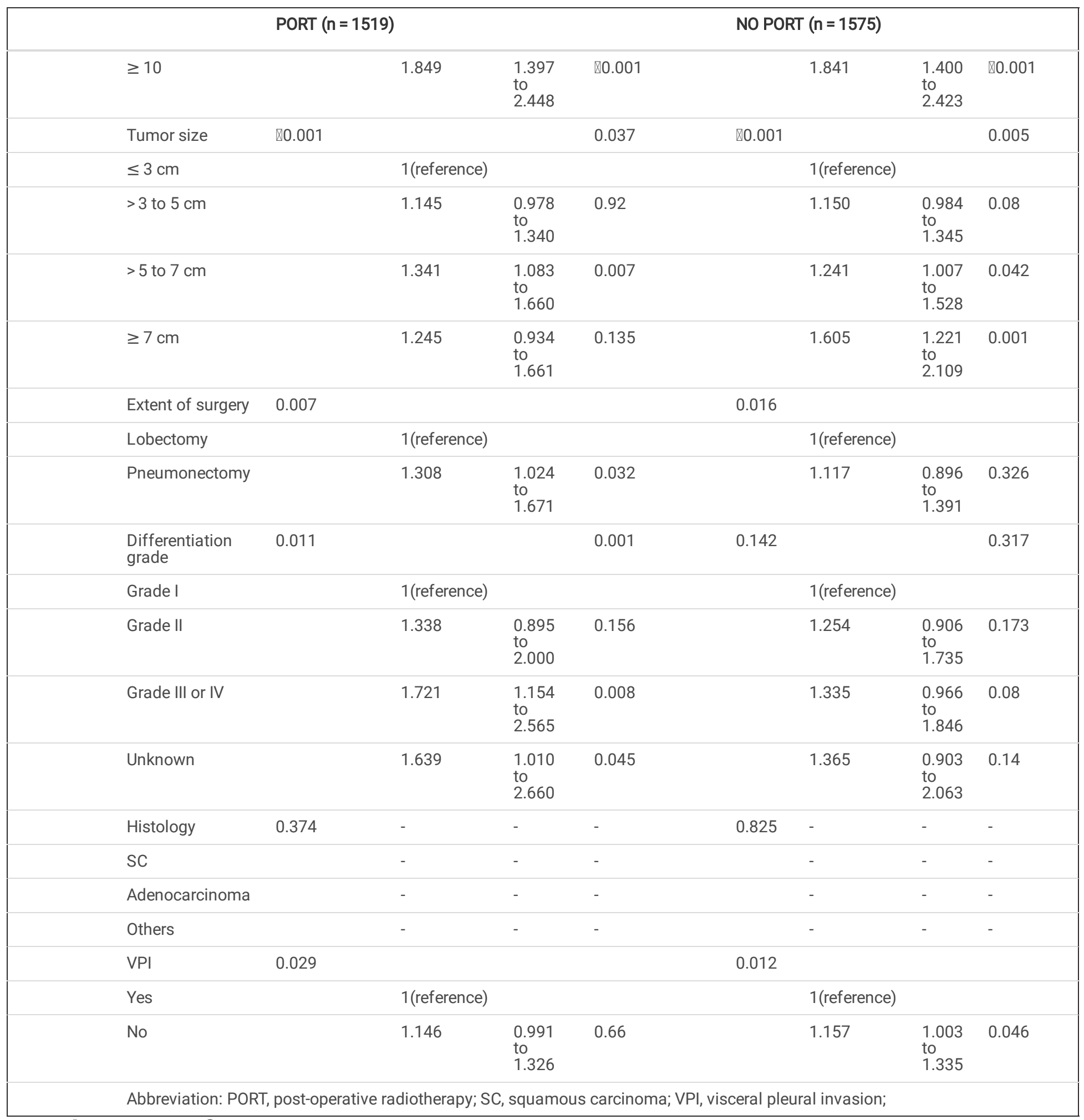

\section{Development of a Prognostic Nomogram}

Nomograms were constructed from the coefficients of the multivariate regression model. Significant independent factors of the two groups, including age, gender, examined lymph node, positive lymph node, tumor size, extent of surgery, differentiation grade, and VPI were included to develop the nomogram. To estimate the net survival differernces for PORT, the two nomograms were utilized together (Fig. 2). The first nomogram (Fig. 2A) estimated OS with PORT, and the second nomogram (Fig. 2B) estimated OS without PORT. The difference between the two estimates represented the expected net survival difference from the addition of PORT. Each factor was given a score on the point scale. By gathering the total score and finding it on the total point scale, it was a simple process to determine the estimated probability of survival at each score point using a straight line. 
The calibration curve (Fig. 3A and B) showed a great agreement between the nomogram prediction and actual observation for one, three, and five year OS in the training cohort. In the PORT group, the Harrell's C-index for the established nomogram to predict OS (0.619; $95 \% \mathrm{Cl}, 0.598$ to 0.641$)$ was significantly greater than that of the IASLC 8th edition staging system (T1, T2, T3 and T4, $0.566 ; 95 \% \mathrm{Cl}, 0.521$ to $0.610 ; \mathrm{P}<0.01)$. In the nonPORT group, the C-index was higher for the nomogram prediction 0.627 (95\% $\mathrm{Cl}, 0.605-0.648)$ than for the $\mathrm{T}$ category prediction ( 0.559 ; $95 \% \mathrm{Cl}$, 0.540 to $0.610 ; P<0.01)$. In the external validation cohort, the calibration plots also presented an acceptable agreement between the nomogram predictions and actual observations for one, three and five year OS (Fig. 3C and D). The C-index was 0.599 (95\% Cl, 0.485 to 0.713$)$ for the PORT group and $0.595(95 \% \mathrm{Cl}, 0.544$ to 0.646$)$ for the non-PORT group.

\section{Clinical Use}

For an individual patient, first use nomogram A to calculate the expected OS with PORT; then use nomogram B to calculate the expected OS without PORT. The difference between the two estimates represents the expected net survival difference from the addition of PORT. The three year survival rates of each patient were calculated using these two nomograms. The net survival difference was computed according to the value of the threeyear survival rates of each patient. There are 1434 patients with a positive PORT net survival difference and 1475 patients with a negative PORT net survival difference for SEER. The multivariate analyses showed that PORT could improve OS (HR, 0.861; 95\% Cl, 0.744 to 0.996; $P=0.044)$ for patients with a positive PORT net survival difference. For patients with a negative PORT net survival difference, PORT was not associated with OS (HR, 1.113; $95 \% \mathrm{Cl}, 0.978$ to $1.267 ; P=0.105)$. The Kaplan-Meier curves for OS are shown in Figure S2.

\section{Discussion}

We established a practical survival prediction model that can be used to make individualized predictions about the expected survival difference of PORT and without PORT for patients with completely resected pathologic N2 NSCLC treated with chemotherapy. The model is more predictive than the T category prediction of the IASLC 8th edition staging manual with higher C-index. The model is practical for individual recommendations for the use of PORT.

As adjuvant chemotherapy is recommended for patients with completely resected pathologic N2, in the NCCN guidelines [3]. Our study excluded patients who had not undertaken chemotherapy. In this large population-based study, our results suggest that there were no statistical difference in OS between the PORT and non-PORT groups (Fig. 1). Similarly, an early closed randomized controlled trial [9] indicated PORT increased both local/regional and distant disease-free survival (DFS) rate, but not the OS rate. A Randomized Phase III study [8] with 37 patients and Phase II trial with 101 patients [10], also showed that there were no statistical differences between the observation and PORT arms for OS. The randomized controlled trial (NCT00410683) announced the results that postoperative radiotherapy has no PFS or OS benefit for R0 resection N2 (IIIA) of NSCLC in the recent ESMO congress. However, previous retrospective studies $[4,5]$ and meta-analyses studies $[6,7]$ revealed that PORT could significantly improve the survival of patients. Therefore, the role of PORT in completely resected N2 patients is still controversial. Our model can provide individual predictions of OS for patients with PORT and non-PORT. This suggests that our nomograms may also be valuable at picking out patients who are most likely to gain from PORT. In addition, we have found that approximately half of the patients may have benefited from PORT (figure S2).

It is unclear why the results of our study were different from others [4, 5], but a possible explanation is the difference in the prognostic factors included in the multivariate analyses. The numbers of positive lymph nodes and examined lymph nodes were not reported in previous NCDB studies $[5,15]$. Especially, the number of positive lymph nodes is an essential prognostic factor in many kinds of cancer, and comparable studies $[16,17]$ also suggested that the higher number of positive lymph nodes $(n>3)$ are associated with a worsening survival rate. In addition, examined lymph node count was an important prognostic factor for NSCLC [18]. The independent prognostic factors consisting of age, gender, tumor size, extent of surgery in our outcomes, were comparable to those discovered in some prior studies for NSCLC [5, 19, 20]. Histology was not selected as a candidate factor because it is not an independent prognostic factor. This finding is consistent with other findings for stage II or III [4] and IIIA-N2 [20] NSCLC from SEER. Furthermore, we found that VPI is associated with poor prognosis in N2 stage NSCLC. The cutoff points are mostly based on our previous studies[18, 19]. A cutoff point of 3 positive lymph nodes was recommended. Therefore, age, sex, examined lymph node, positive lymph node, tumor size, extent of surgery, differentiation grade, and VPI were the final factors applied in the nomogram.

The calibration plots in the training and external validation cohorts indicated ideal agreement between actual OS and prediction, indicating that predictive functionality of the nomograms was excellent. The C-indices from our nomograms ( 0.63 and 0.66 for PORT and non-PORT cohorts, respectively) were superior to that of TNM staging ( 0.56 and 0.55$)$, with $P<.001$ in OS. Therefore, by combining multiple clinical risk factors, the nomogram had a better discrimination ability than the TNM staging system. Moreover, the nomograms can be generally applied considering that the data gathered from the United States multi-center SEER database and the two centers in China might reduce the impact of patient history backgrounds and hospital differences.

Despite there being several NSCLC prognostic models had been reported previously[18-22], no nomogram has been developed for completely resected pathologic N2 NSCLC with and without PORT. We previously developed a survival model to predict OS for patients with stage I- IIIA resected NSCLC, but it only included $24 \%$ of N2 patients and was not related to PORT [18]. A recent study [20] reported a nomogram to predict the

Page $8 / 13$ 
survival of stage IIIA-N2 NSCLC after surgery. However, it lacked chemotherapy data and cannot guide the choice of PORT. Jiang et al.[23] reported a similar survival prediction model in patients with stage II or III gastric cancer. Their nomgrams can be applied to calculate individualized predictions of the probable OS advantage from adjuvant chemotherapy for these patients. We also established practical nomograms to predict OS and identified a subset of patients who might benefit from PORT.

There remain some limitations to our study. Firstly, the clinical characteristics of patients with and without PORT were slightly different between the training and external validation cohorts (Table 1), especially the proportion and number of PORT in the Chinese patients was very low. Therefore, our results should be further validated by larger multi-center data from other countries. Secondly, our study is limited by its retrospective design, which represents an unavoidable bias. Furthermore, the SEER program lacks data on some relevant molecular factors, chemotherapy regimen, tumor recurrence, RT dose, surgical margin status, and comorbidity. In addition, the C-indices of the nomogram were only 0.619 and 0.627 , which was not inspiring. Obviously, future studies using prospective data collection and additional prognostic variables are needed to improve performance and reliability of the model.

\section{Conclusion}

We established a practical nomogram that can produce an individualized estimate of the net survival difference of PORT and without PORT for patients with completely resected pathologic N2 NSCLC treated with chemotherapy. This model can help us to quantify the survival benefit of PORT after surgical resection of N2 NSCLC with chemotherapy and to make individualized therapeutic suggestions.

\section{Abbreviations}

Non-small-cell lung cancer (NSCLC); post-operative radiotherapy (PORT); Overall survival (OS); National Comprehensive Cancer Network (NCCN); Surveillance, Epidemiology, and End Results (SEER); Visceral pleural invasion (VPI); Confidence interval (CI); Interquartile range (IQR)

\section{Declarations}

\section{Ethics approval and consent to participate}

The study was approved by the First Affiliated Hospital of Guangzhou Medical University, and informed consent was waived because of the retrospective nature of this study.

\section{Consent for publication}

Not applicable

\section{Availability of data and material}

The datasets analyzed during the current study are available from the corresponding author on reasonable request

\section{Competing interests}

We have no conflicts of interest to declare.

\section{Funding}

This study was supported by the China State Key Laboratory of Respiratory Disease Independent Subject (Grant No. SKLRD-QN-201925); Guangdong Provincial Basic and Applied Basic Research Fund of China (Grant No. 2020A1515110445) $₫$ National key R \& D Program of China (Grant No. 2017YFC0907903 \& 2017YFC0112704).

\section{Authors' contributions}

Conception and design: Jianxing He, Yuan Zeng, Wenhua Liang, Jun Liu

Collection and assembly of data: Jianxing He, Yuan Zeng, Wenhua Liang, Qiwen Li, Hui Liu, Minghui Wan

Data analysis and interpretation: All authors

Manuscript preparation: All authors

Manuscript proofing: All authors

Final approval of manuscript: All authors

\section{Acknowledgements}


Not applicable

\section{References}

1. Bray F, Ferlay J, Soerjomataram I, Siegel RL, Torre LA, Jemal A. Global cancer statistics 2018: GLOBOCAN estimates of incidence and mortality worldwide for 36 cancers in 185 countries. CA Cancer J Clin. 2018;68:394-424.

2. Betticher DC, Hsu Schmitz SF, Tötsch M, Hansen E, Joss C, von Briel C, et al. Prognostic factors affecting long-term outcomes in patients with resected stage IIIA pN2 non-small-cell lung cancer: 5-year follow-up of a phase II study. Br J Cancer. 2006;94:1099-106.

3. National Comprehensive Cancer Network: NCCN Clinical Practice Guidelines in Oncology: NonSmall Cell Lung Cancer. V. 1.2017. www.nccn.org/professionals/physician_gls/PDF/nscl.pdf. .

4. Lally BE, Zelterman D, Colasanto JM, Haffty BG, Detterbeck FC, Wilson LD. Postoperative radiotherapy for stage II or III non-small-cell lung cancer using the surveillance, epidemiology, and end results database. J Clin Oncol. 2006;24:2998-3006.

5. Robinson CG, Patel AP, Bradley JD, DeWees T, Waqar SN, Morgensztern D, et al. Postoperative radiotherapy for pathologic N2 non-small-cell lung cancer treated with adjuvant chemotherapy: a review of the National Cancer Data Base. J Clin Oncol. 2015;33:870-6.

6. Sakib N, Li N, Zhu X, Li D, Li Y, Wang H. Effect of postoperative radiotherapy on outcome in resectable stage IIIA-N2 non-small-cell lung cancer: an updated meta-analysis. Nucl Med Commun. 2018;39:51-9.

7. Billiet C, Decaluwé H, Peeters S, Vansteenkiste J, Dooms C, Haustermans K, et al. Modern post-operative radiotherapy for stage III non-small cell lung cancer may improve local control and survival: a meta-analysis. Radiother Oncol. 2014;110:3-8.

8. Perry MC, Kohman LJ, Bonner JA, Gu L, Wang X, Vokes EE, et al. A phase III study of surgical resection and paclitaxel/carboplatin chemotherapy with or without adjuvant radiation therapy for resected stage III non-small-cell lung cancer: Cancer and Leukemia Group B 9734 . Clin Lung Cancer. 2007;8:268-72.

9. Shen WY, Ji J, Zuo YS, Pu J, Xu YM, Zong CD, et al. Comparison of efficacy for postoperative chemotherapy and concurrent radiochemotherapy in patients with IIIA-pN2 non-small cell lung cancer: an early closed randomized controlled trial. Radiother Oncol. 2014;110:1205 .

10. Sun JM, Noh JM, Oh D, Kim HK, Lee SH, Choi YS, et al. Randomized Phase II Trial Comparing Chemoradiotherapy with Chemotherapy for Completely Resected Unsuspected N2-Positive Non-Small Cell Lung Cancer. J Thorac Oncol. 2017;12:1806-13.

11. Goldstraw P, Chansky K, Crowley J, Rami-Porta R, Asamura H, Eberhardt WE, et al. The IASLC Lung Cancer Staging Project: Proposals for Revision of the TNM Stage Groupings in the Forthcoming (Eighth) Edition of the TNM Classification for Lung Cancer. J Thorac Oncol. 2016;11:3951.

12. Frank E HJR. Regression Modeling Strategies. R Package version 3.4-0. http://www.rproject.org/. .

13. Iasonos A, Schrag D, Raj GV, Panageas KS. How to build and interpret a nomogram for cancer prognosis. J Clin Oncol. 2008;26:1364-70.

14. Hanley JA, McNeil BJ. A method of comparing the areas under receiver operating characteristic curves derived from the same cases.

Radiology. 1983;148:839-43.

15. Wong AT, Rineer J, Schwartz D, Schreiber D. Assessing the Impact of Postoperative Radiation Therapy for Completely Resected LimitedStage Small Cell Lung Cancer Using the National Cancer Database. J Thorac Oncol. 2016;11:242-8.

16. Fukui T, Mori S, Yokoi K, Mitsudomi T. Significance of the number of positive lymph nodes in resected non-small cell lung cancer. J Thorac Oncol. 2006;1:120-5.

17. Shang X, Liu J, Li Z, Lin J, Wang H. A hypothesized TNM staging system based on the number and location of positive lymph nodes may better reflect the prognosis for patients with NSCLC. BMC Cancer. 2019;19:591.

18. Liang W, Zhang L, Jiang G, Wang Q, Liu L, Liu D, et al. Development and Validation of a Nomogram for Predicting Survival in Patients With Resected Non-Small-Cell Lung Cancer. J Clin Oncol. 2015.

19. Zeng Y, Mayne N, Yang CJ, D'Amico TA, Ng C, Liu CC, et al. A Nomogram for Predicting Cancer-Specific Survival of TNM 8th Edition Stage I Non-small-cell Lung Cancer. Ann Surg Oncol. 2019. 
20. Mao Q, Xia W, Dong G, Chen S, Wang A, Jin G, et al. A nomogram to predict the survival of stage IIIA-N2 non-small cell lung cancer after surgery. J Thorac Cardiovasc Surg. 2018;155:1784-92.e3.

21. Kratz JR, He J, Van Den Eeden SK, Zhu ZH, Gao W, Pham PT, et al. A practical molecular assay to predict survival in resected non-squamous, non-small-cell lung cancer: development and international validation studies. Lancet. 2012;379:823-32.

22. Birim O, Kappetein AP, Waleboer M, Puvimanasinghe JP, Eijkemans MJ, Steyerberg EW, et al. Long-term survival after non-small cell lung cancer surgery: development and validation of a prognostic model with a preoperative and postoperative mode. J Thorac Cardiovasc Surg. 2006;132:491-8.

23. Jiang Y, Li T, Liang X, Hu Y, Huang L, Liao Z, et al. Association of Adjuvant Chemotherapy With Survival in Patients With Stage II or III Gastric Cancer. JAMA Surg. 2017;152:e171087.

\section{Figures}
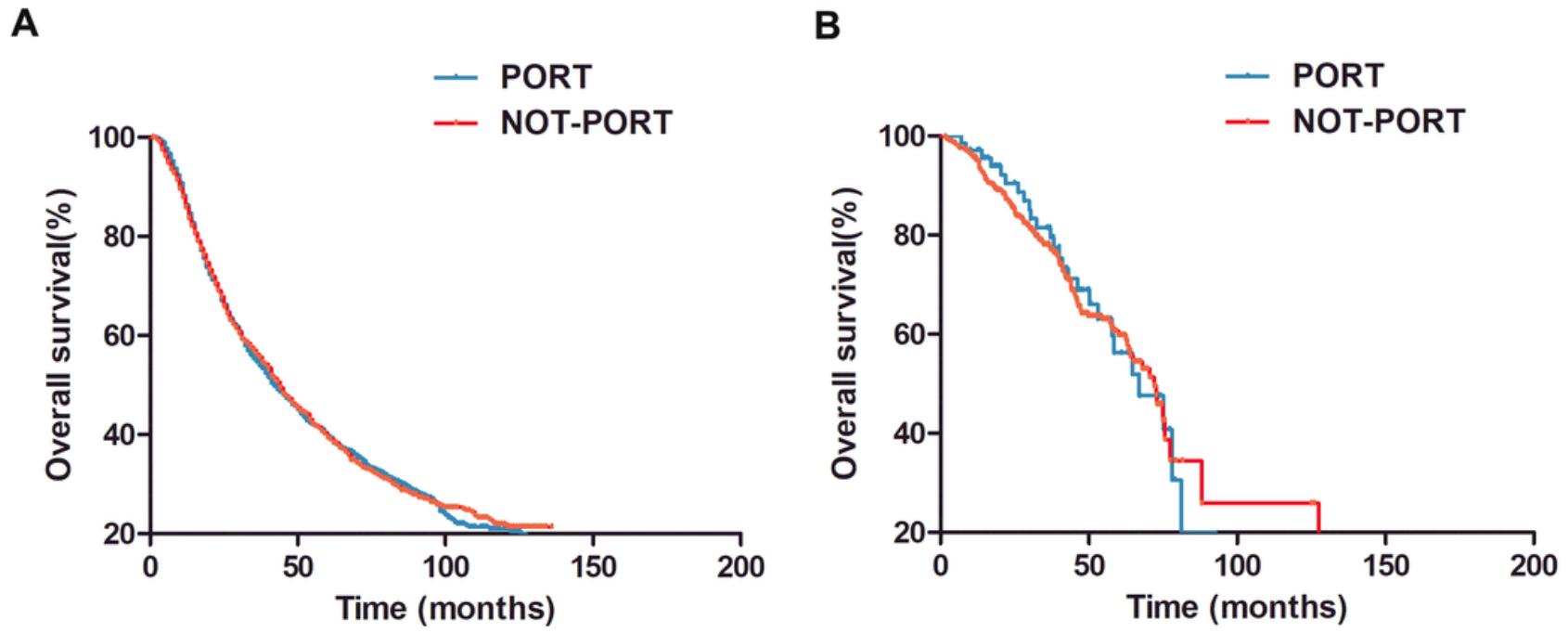

Figure 1

Kaplan-Meier Estimates of Overall Survival (OS) for all patients in the training (A) and external validation cohorts (B). 


\section{A Expected OS with PORT}

Points

Age

Gender

Examined lymph node

Positive lymph node

Extent of surgery

Tumor size

Differentiation

Total Points

1-year survival

3-year survival

5-year survival
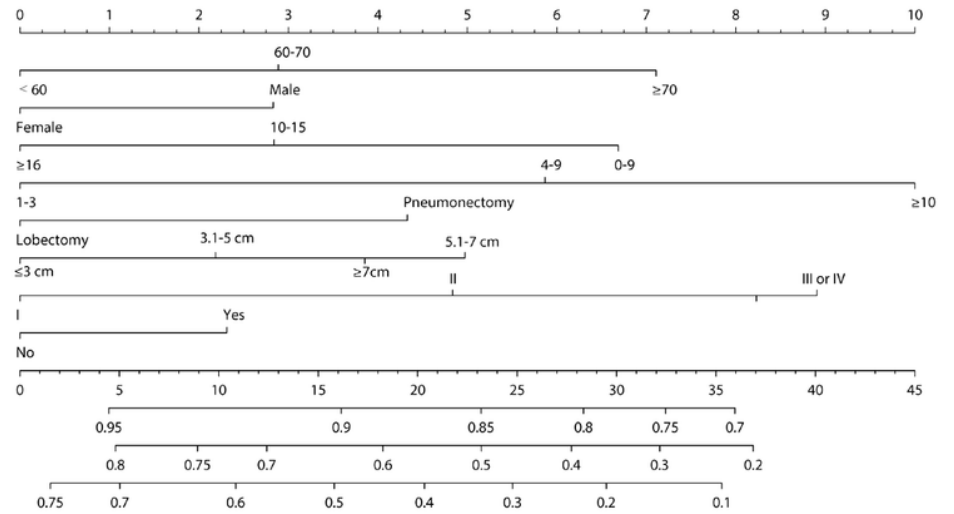

\section{B Expected OS witout PORT}

Points

Age

Gender

Examined lymph node

Positive lymph node

Extent of surgery

Tumor size

Differentiation

VPI

Total Points

1-year survival

3-year survival

5-year survival

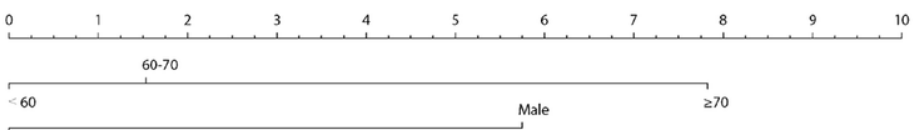

Female 10-15

$\geq 16 \quad 0.9 \quad 4-9$

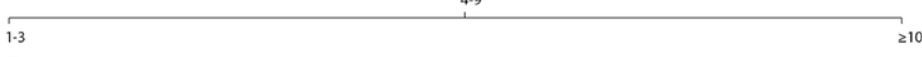

Lobectomy and pneumonectomy $3.1 .5 \mathrm{~cm}$

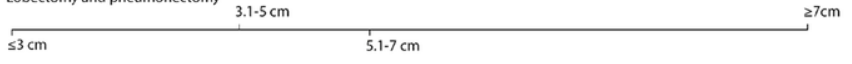

Áll

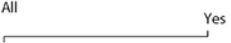

No

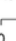

es

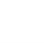

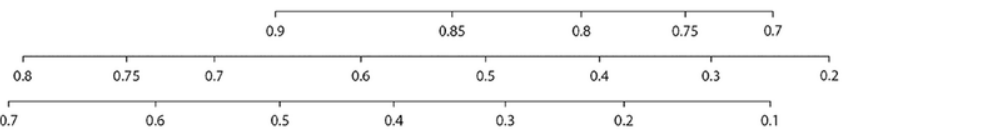

Figure 2

Nomograms for the Comparison of Expected Overall Survival (OS) With and Without post-operative radiotherapy (PORT). For an individual patient, first use nomogram A to calculate the expected OS with PORT; then use nomogram B to calculate the expected OS without PORT. The difference between the two estimates represents the expected net survival impact from PORT and without PORT. PORT, post-operative radiotherapy; VPI, visceral pleural invasion. 
A

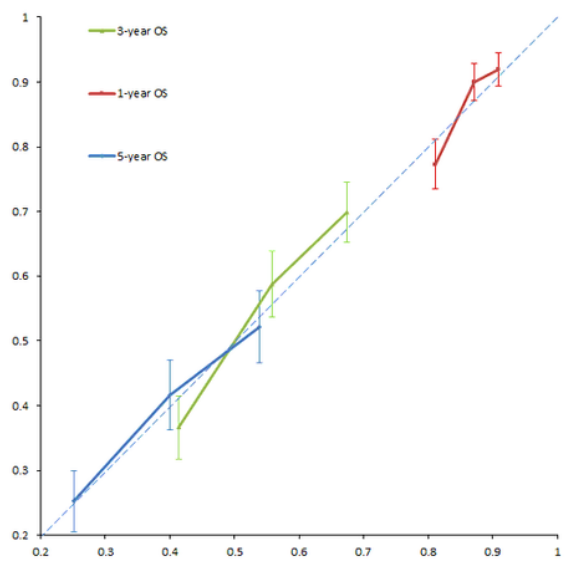

C

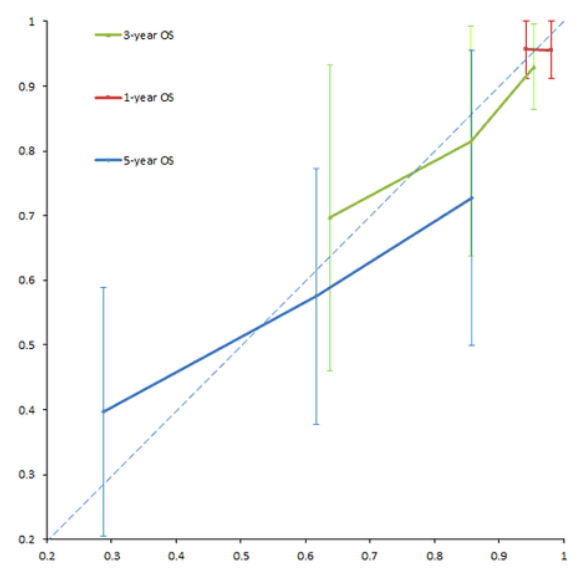

B

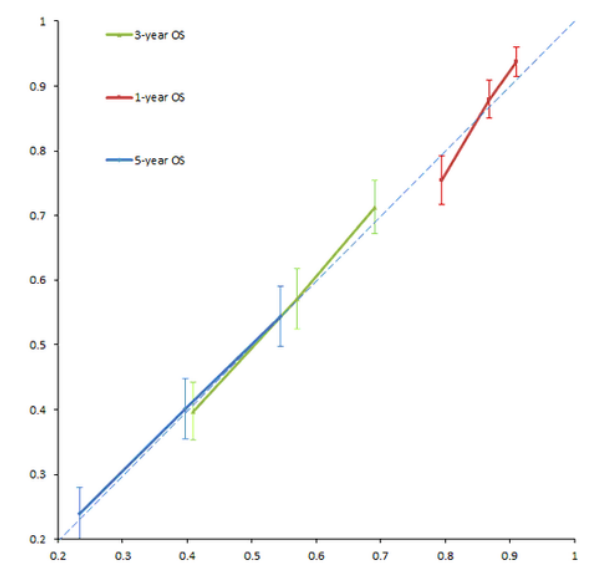

D

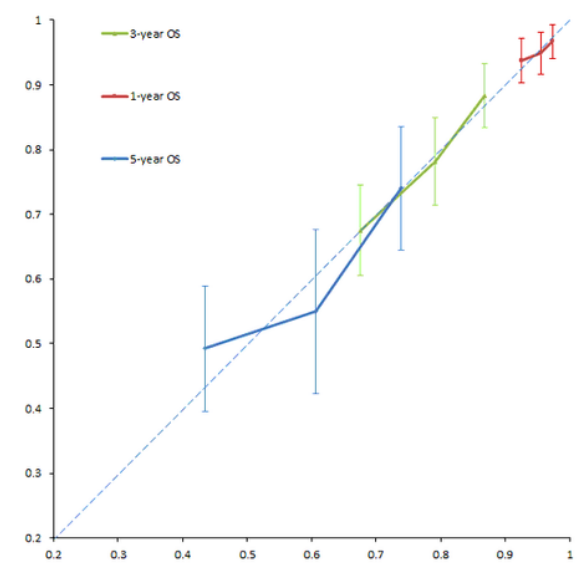

\section{Figure 3}

Calibration of the Nomograms in the Training and External Validation Cohorts. The $x$-axis represents the nomogram-predicted survival, the $y$-axis represents actual survival, and $95 \% \mathrm{Cls}$ are measured by Kaplan-Meier analysis. Calibration curves of PORT (A) and non-PORT (B) group in the training cohort. Calibration curves of PORT (C) and non-PORT (D) group in the external validation cohort.

\section{Supplementary Files}

This is a list of supplementary files associated with this preprint. Click to download.

- figureS1.tif

- figureS2.tif 